\title{
Preliminary exploration on the role of clinical pharmacists in cancer pain pharmacotherapy
}

\author{
Ying-Jie Su ${ }^{1,2 \#}$, Yi-Dan Yan ${ }^{1 \#}$, Wen-Juan Wang ${ }^{3}$, Tao Xu ${ }^{4}$, Zhi-Chun Gu ${ }^{1}$, Yong-Rui Bai ${ }^{2}$, Hou-Wen Lin ${ }^{1}$ \\ ${ }^{1}$ Department of Pharmacy, ${ }^{2}$ Department of Radiation Oncology, Renji Hospital, School of Medicine, Shanghai Jiaotong University, Shanghai, China; \\ ${ }^{3}$ Department of Pharmacy, Children's Hospital of Soochow University, Suzhou, China; ${ }^{4}$ Department of Pharmacy, Ningbo First Hospital, Ningbo, \\ China \\ Contributions: (I) Conception and design: YJ Su, YD Yan; (II) Administrative support: ZC Gu, HW Lin; (III) Provision of study materials or patients: \\ YJ Su, YD Yan; (IV) Collection and assembly of data: YJ Su, YD Yan; (V) Data analysis and interpretation: YJ Su, YD Yan, ZC Gu; (VI) Manuscript \\ writing: All authors; (VII) Final approval of manuscript: All authors. \\ \#These authors contributed equally to this work as co-first authors. \\ Correspondence to: Zhi-Chun Gu. Department of Pharmacy, Renji Hospital, School of Medicine, Shanghai Jiao Tong University, Shanghai, China; \\ Email: guzhichun213@163.com; Yong-Rui Bai. Department of Radiation Oncology, Renji Hospital, School of Medicine, Shanghai Jiao Tong \\ University, Shanghai, China. Email: baiyongrui@renji.com.
}

Background: More than half of cancer patients affected by cancer experience pain of moderate-tosevere intensity. Therefore, facilitating appropriate and safe administration of analgesics is crucial to the comprehensive management of cancer patients. In this article, we assessed medication adherence, pain relief, drug related problems (DRPs) and analgesics adverse events (AEs) in cancer pain patients based on a model of clinical pharmacy services.

Methods: In this prospective, single-arm intervention study, cancer pain patients admitted to our institution were eligible. According to different adherence, heterogeneity of pain, and individual treatment strategy, clinical pharmacists (CPs) provided comprehensive pain assessment and medication education for patients, as well as provided consultation and recommendation for physicians. CPs' pharmacy services were assessed through medication adherence, numbers of DRPs, acceptance of recommendation, pain intensity (PI), daily interference and AEs.

Results: A total of 42 patients were enrolled between November, 2018 and November, 2019. Compared to baseline, patients' medication adherence evaluated with a medication adherence scale showed a significantly improvement at 14 and at 28 days after receiving CPs' interventions (8 score $v s$. 7 score at 14 days and at 28 days, $\mathrm{P}<0.01)$. During the 28 -day follow-up, a total of 63 interventions were put forward according to 57 identified DRPs in 33 patients (78.6\%), and approximately $95 \%(60 / 63)$ of the interventions were accepted by physicians. PI and daily interference significantly improved on the third day after the interventions of CPs, and the improvement continued until day $28(\mathrm{P}<0.01)$. AEs caused by opioids occurred in 19 patients $(45.2 \%)$, and the most common one was constipation (14 patients, $33.3 \%)$.

Conclusions: CPs' comprehensive interventions for cancer pain patients were efficacious in improving their medication adherence and pain relief, as well as reducing incidence of AEs. Therefore, this promising model should be replicated in other medical centers.

Keywords: Cancer pain; clinical pharmacist (CP); pharmacy services; adherence; drug related problems (DRPs)

Submitted Mar 12, 2020. Accepted for publication Jun 23, 2020.

doi: $10.21037 /$ apm-20-627

View this article at: http://dx.doi.org/10.21037/apm-20-627 


\section{Introduction}

Cancer is the leading cause of death in China with increasing incidence and mortality, and is a major public health problem (1). In oncological population, pain remains a common and distressing symptom, affecting approximately $59 \%$ of patients undergoing anticancer treatment, $64 \%$ of patients with advanced/metastatic cancer, and $33 \%$ of patients having completed curative treatment $(2,3)$. The guidelines for the management of cancer pain were developed by the World Health Organization (WHO) in 1986 , but there is substantial evidence that the management of cancer pain is still often suboptimal. Barriers to adequate pain management include societal attitudes toward pain management, system and regulatory barriers, clinician barriers, patient barriers, as well as racial and socioeconomic disparities in the assessment and management of pain (4). One of the most pervasive barriers in clinician is inadequate provider knowledge regarding pain management $(3,4)$. A survey in China showed that medical school/residency training with regard to cancer pain management was inadequate in $80 \%$ of physicians, because of little formal time for pain management education throughout their training and career (5). With regard to patient-related barriers, worry about side effects, fear of addiction and reluctance to report pain are primary ones (5). For above reasons, it is essential to shed light on a new model of management to improve cancer pain pharmacotherapy for Chinese population.

Of late years, clinical pharmacists (CPs) play an increasingly important role in pharmacotherapy management in China (6,7). CPs have carried out a lot of clinical pharmacy services to promote rational use of medicines in cancer pain patients by comprehensively evaluating cancer pain, giving suggestions on drug treatment to physicians, and conducting pharmaceutical education to patients (7). According to a Chinese study, participation by the pharmacist in the cancer pain multidisciplinary management team led to a marked reduction in most of the drug-related problems (DRPs) and a statistically significant change in pain score (8). Moreover, Zhai et al. suggested that detecting and correcting DRPs by CPs brought about positive effects in reducing mortality in patients with acute myocardial infarction (6), while this pattern has not been evaluated in patients with cancer pain. Thus, this study aims to assess medication adherence, pain relief, DRPs and analgesics adverse events (AEs) in cancer pain patients based on a model of clinical pharmacy services. We present the following article in accordance with the
STROBE reporting checklist (available at http://dx.doi. org/10.21037/apm-20-627).

\section{Methods}

\section{Study design and patient population}

This study is a prospective, single-arm intervention trial done in a teaching hospital (Renji Hospital, School of Medicine, Shanghai Jiaotong University) between November, 2018 and November, 2019. The CP participated in daily ward rounds with physicians in department of radiation oncology, and new admitted patients were eligible for inclusion if they met the following criteria: (I) aged 18 years or older; (II) confirmed diagnosis of cancer; (III) diagnosis of nociceptive pain related to cancer or cancer therapy by the treating physician; (IV) life expectancy of at least 3 months; (V) able to comprehend, speak, and read Chinese. Patients were excluded in case of invasive pain treatment (e.g., nerve block or patient-controlled analgesia). The study was conducted in accordance with the Declaration of Helsinki (as revised in 2013). This study was approved by the Ethics Committee of Renji Hospital, School of Medicine, Shanghai Jiaotong University (KY2020-036). Written informed consent was obtained from the patients for publication of this study.

\section{Procedure and intervention}

The CP working in radiation oncology department is skilled in pharmacotherapy management of cancer pain patients for 10 years working experience. In the course of CP's intervention, face-to-face interviews were conducted with enrolled patients. At study entry, patients received a comprehensive assessment for medication adherence (9) and cancer pain, including pain management history, pain characteristics (site, description, timing, etc.), pain intensity (PI) (worst pain, least pain, average pain in last 24 hours and current pain), interference for daily life, current analgesic strategy, pain relief and adverse effects. The project was carefully designed in accordance with the National Comprehensive Cancer Network Adult Cancer Pain Guidelines (version 1.2018). Afterwards, the CP provided personalized medication education according to their medication adherence. For patients with good medication adherence (score $\geq 6$ ), we gave them a 10 -minutes face-toface medication education as well as an "education manual for pain patients" for reading by themselves. For those 
with weak medication adherence (score $<6$ ), we spent about 20 minutes for medication education, including detailed explaining the "education manual for pain patients". After initial medication education, reassessment of medication adherence, PI and daily interference were done based on the project (medication adherence: at day 14 and 28; PI and daily interference: at day 3, 7, 14 and 28). During 28-day follow-up, patients were monitored for analgesic efficacy and safety every day during hospitalization, and were scheduled for receiving medication education and reassessment of cancer pain via telephone weekly after discharge. In addition, possible DRPs were identified by the CP based on her daily ward rounds with physicians, examination of prescriptions, and patient-pharmacist interview. Accordingly, advices to optimize analgesic therapy were offered for physicians. All the DRPs and recommendations were documented, whether or not the physician chose to accept them.

\section{Data collection}

The study data were collected from inpatient records, face-to-face meeting and interviews via telephone. Patient characteristics were recorded at study entry. Health-related variables included Eastern Cooperative Oncology Group Performance Status (ECOGPS), cancer type and tumor stage (locally advanced disease or metastatic disease). The medication adherence of patients was evaluated with an 8 -item medication adherence scale. Numerical rating scales (NRS), one of the most frequent methods for PI assessment, was used to measure the worst pain, least pain and average pain in the last 24 hours, as well as pain right now. Painrelated interference of daily life (daily interference) was assessed through 7 items (general activity, mood, walking ability, normal work, sleep, relations with other people and enjoyment of life) (10). Meanwhile, pain information, initial analgesic drug, morphine milligram equivalents (MMEs), AEs, drug related problems (DRPs) and recommendations provided by $\mathrm{CP}$ during the course of treatment were also recorded.

\section{Outcome assessment}

The impact of CPs' interventions was assessed by: (I) medication adherence; (II) the number of DRPs and acceptance of recommendation; (III) PI and daily interference; (IV) AEs of analgesics.

\section{Data analysis}

Categorical variables are expressed as the number and percentage and compared by Fisher's exact test or Chisquare test. Continuous variables are expressed as mean with standard deviation or median with quartile, and compared by paired student's $t$-test or ANOVA test, as appropriate. All statistical analyses were performed using IBM SPSS version23 (IBM Corp., Armonk, NY, USA) and $\mathrm{P}<0.05$ indicated a statistically significant difference.

\section{Results}

\section{Patient characteristics}

Totally, 42 patients were enrolled between November, 2018 and November, 2019. Two patients on day 14 and six patients on day 28 had discontinued during followup because of death or withdrawal of analgesic. Baseline characteristics of the 42 patients who received interventions are summarized in Table 1. The mean age of patients was 59 years, the percentage of male was 66.7 and most of them were graduated from high school $(73.8 \%)$. The $90.5 \%$ of patients were diagnosed with metastatic cancer, and the top three types of cancer were esophageal carcinoma (21.4\%), lung cancer (19.0\%) and cervical cancer (19.0\%), respectively.

Baseline pain-related characteristics are presented in Table 2. Only one patient had more than one site of pain at baseline. As for pain location, bone pain, somatic pain and visceralgia accounted for $33.3 \%, 31 \%$ and $28.6 \%$, respectively. In terms of initial strategy, half of patients were prescribed oxycodone sustained-release tablet, and three patients received inappropriate analgesics (fentanyl transdermal patch for opioid-naive patient; oxycodone and acetaminophen tablets as initial analgesics; morphine hydrochloride injection to deal with breakthrough pain for patient who can take oral medications).

The daily MMEs per patient was $59.82 \pm 64.50$ at baseline, and were $70.36 \pm 48.66,73.93 \pm 55.88,80.88 \pm 64.28$ and $78.06 \pm 74.85$ at $3,7,14$ and 28 days after CP interventions, respectively.

\section{Medication adberence}

After CP interventions, medication adherence scale scores showed a significantly improvement compared to baseline (8 score $v s .7$ score at 14 days and at 28 days, $\mathrm{P}<0.01)$. Of note, 
Table 1 Baseline socio-demographics and clinical characteristics

\begin{tabular}{|c|c|}
\hline Parameters & Patients number $(n=42)$ \\
\hline Age, mean years [SD] & 59 [10] \\
\hline Gender, male & $28(66.7 \%)$ \\
\hline \multicolumn{2}{|l|}{ Education completed } \\
\hline Primary/elementary school & $9(21.4 \%)$ \\
\hline High school & $31(73.8 \%)$ \\
\hline Bachelor's degree or above & $2(4.8 \%)$ \\
\hline \multicolumn{2}{|l|}{ ECOG PS } \\
\hline 0 & $1(2.4 \%)$ \\
\hline 1 & $20(47.6 \%)$ \\
\hline 2 & $10(23.8 \%)$ \\
\hline 3 & $11(26.2 \%)$ \\
\hline \multicolumn{2}{|l|}{ Tumor types } \\
\hline Esophageal carcinoma & $9(21.4 \%)$ \\
\hline Lung cancer & $8(19.0 \%)$ \\
\hline Cervical cancer & $8(19.0 \%)$ \\
\hline pancreatic cancer & $6(14.3 \%)$ \\
\hline Others & $11(26.2 \%)$ \\
\hline \multicolumn{2}{|l|}{ Tumor stage } \\
\hline Locally advanced cancer & $4(9.5 \%)$ \\
\hline Metastatic cancer & $38(90.5 \%)$ \\
\hline
\end{tabular}

ECOG PS, Eastern Cooperative Oncology Group Performance Status.

8 patients had a poor medication adherence at baseline, with medication adherence scale scores below than 6 , while no patient with a poor medication adherence was observed at 14 and 28 days after $\mathrm{CP}$ interventions.

\section{DRPs and interventions from $C P$}

A total of 57 DRPs were identified in 33 patients (78.6\%) and more than 1 DRP was identified in 17 patients (2 DRPs in 11 patients, 3 DRPs in 4 patients and 4 DRPs in 2 patients). Accordingly, the CP put forward 63 recommendations for physicians, and approximately $95 \%$ of them (60/63) were accepted. Details of DRPs and recommendations are listed in Table 3. Inadequate pain control was considered to be the primary DRP, and the CP provided different suggestions individually, including
Table 2 Baseline pain-related characteristics

\begin{tabular}{lc}
\hline Parameters & Patients number $(\mathrm{n}=42)$ \\
\hline Number of pain locations & $41(97.6 \%)$ \\
1 & $1(2.4 \%)$ \\
2 & \\
Pain sites & $14(33.3 \%)$ \\
Bone pain & $13(31.0 \%)$ \\
Somatic pain & $12(28.6 \%)$ \\
Visceral pain & $4(9.5 \%)$ \\
Neuropathic pain & \\
Initial analgesics & $21(50 \%)$ \\
Oxycodone sustained-release & \\
tablets & $12(28.6 \%)$ \\
Morphine sustained-release & \\
tablets & $5(11.9 \%)$ \\
Tramadol sustained-release & $1(2.4 \%)$ \\
tablets & \\
Fentanyl transdermal patches & \\
Oxycodone and Acetaminophen \\
tablets \\
$\begin{array}{l}\text { Morphine tablets } \\
\text { lbuprofen capsules }\end{array}$ & \\
\hline & $1(2.4 \%)$ \\
\hline
\end{tabular}

short-acting opioids administration (16, 25.4\%), longacting opioids increase (13,20.6\%), adjuvant analgesics combination $(8,12.7 \%)$ and opioids initiation $(4,6.3 \%)$. Of note, two patients experienced end-of-dose failure pain, which recurred towards the end of dosing interval for regularly scheduled opioids. Therefore, $\mathrm{CP}$ recommended physicians increase the frequency of regularly scheduled opioids rather than dosage to achieve pain relief.

\section{PI and daily interference}

Both PI and daily interference were ameliorated significantly on the third day after the intervention by $\mathrm{CP}(\mathrm{P}<0.01)$, and this improvement continued to the end of 28-day follow-up (Table 4).

\section{$A E$}

A total of 19 (45.2\%) of 42 patients had opioid-related AEs. The most common AE was constipation, happened 
Table 3 DRPs and interventions from CP

\begin{tabular}{lc}
\hline Parameters & Patients number \\
\hline DRPs ( $\mathrm{n}=57)$ & $36(63.2 \%)$ \\
Inadequate pain control & $9(15.8 \%)$ \\
No attention paid to AE & $8(14.0 \%)$ \\
No opioids dosage reduction in time or & \\
opioids overdose & $3(5.3 \%)$ \\
Inappropriate drugs & $1(1.8 \%)$ \\
AE persist & $16(25.4 \%)$ \\
Interventions from pharmacists ( $\mathrm{n}=63)$ & $13(20.6 \%)$ \\
Short-acting opioids administration & $8(12.7 \%)$ \\
Increasing the dose or frequency of long- & \\
acting opioids & $4(6.3 \%)$ \\
Combined with adjuvant analgesics & $9(14.3 \%)$ \\
Initiating opioids & $8(12.7 \%)$ \\
Combined with medicines to relieve AE & $3(4.8 \%)$ \\
Decreasing the dose or withdrawal \\
opioids \\
$\begin{array}{l}\text { Switch to other medications } \\
\text { Opioids rotation }\end{array}$ \\
\hline $\begin{array}{l}\text { DRPs, drug related problems; CP, clinical pharmacist; AE, adverse } \\
\text { events. }\end{array}$
\end{tabular}

in 14 patients. Others were nausea (6 patients), dysuria (3 patients) and vomit (1 patient) and drowsiness (1 patient). Most of the AEs were resolved except for 2 cases of constipation and 1 case of dysuria.

\section{Discussion}

In the present study, we evaluated the role of CPs in the management of pharmacotherapy for cancer pain patients by focusing on pain assessment, medicine education, as well as recommendations for dealing with DRPs and AEs. The results showed that CPs' pharmacy services significantly improved medication adherence, PI and daily interference in patients with cancer pain.

In China, CPs have been playing an increasingly important role in the management of complex medicine treatment in cancer pain patients, while few studies have specifically addressed in this field. Several previous studies focused on educational interventions $(11,12)$ or DRPs from CP $(8,13)$. Fewer studies, just like ours, tried to demonstrate multifaceted role of CPs in the management of cancer pain $(7,14)$.

According to previous research, CP-led medication education resulted in improved pain control, reduced PI scores and daily interference in patients with cancer (11).

Table 4 Pain intensity and daily interference change

\begin{tabular}{|c|c|c|c|c|c|}
\hline Items & Baseline & Day 3 & Day 7 & Day 14 & Day 28 \\
\hline Worst $\mathrm{PI}$ in last $24 \mathrm{~h}$ & $6.12 \pm 2.33$ & $3.05 \pm 1.71$ & $3.05 \pm 2.27^{*}$ & $2.27 \pm 1.73$ & $2.22 \pm 1.69^{*}$ \\
\hline Least $\mathrm{Pl}$ in last $24 \mathrm{~h}$ & $1.69 \pm 1.73$ & $0.43 \pm 0.94^{*}$ & $0.33 \pm 0.57^{\star}$ & $0.20 \pm 0.51^{*}$ & $0.16 \pm 0.44$ \\
\hline Average $\mathrm{PI}$ in last $24 \mathrm{~h}$ & $3.96 \pm 1.77$ & $1.85 \pm 1.29^{*}$ & $1.70 \pm 1.36^{*}$ & $1.26 \pm 0.99$ & $1.14 \pm 0.95^{*}$ \\
\hline \multicolumn{6}{|l|}{ Daily interference (mean \pm SD) } \\
\hline General activity & $5.40 \pm 2.98$ & $3.31 \pm 2.44^{*}$ & $3.10 \pm 2.55^{\star}$ & $2.32 \pm 2.12^{*}$ & $2.22 \pm 2.12^{*}$ \\
\hline Mood & $5.10 \pm 3.05$ & $2.21 \pm 2.24^{*}$ & $1.86 \pm 2.27^{\star}$ & $1.46 \pm 1.91$ & $1.32 \pm 1.84^{*}$ \\
\hline Walking ability & $5.05 \pm 3.38$ & $2.76 \pm 2.83^{*}$ & $2.43 \pm 2.70^{\star}$ & $1.78 \pm 2.30^{*}$ & $1.68 \pm 2.29^{*}$ \\
\hline Sleep & $5.19 \pm 2.93$ & $2.33 \pm 2.18$ & $1.98 \pm 2.39$ & $1.24 \pm 1.64$ & $1.05 \pm 1.56$ \\
\hline Enjoyment of life & $4.83 \pm 3.48$ & $2.69 \pm 3.02^{*}$ & $2.26 \pm 3.03^{*}$ & $1.56 \pm 2.25^{*}$ & $1.65 \pm 2.29^{*}$ \\
\hline
\end{tabular}

${ }^{*}, \mathrm{P}<0.01$ compared with baseline. PI, pain intensity. 
A systematic review and meta-analysis also demonstrated that pharmacist educational interventions for patients with cancer pain had been found to show promise in reducing PI (12). However, medication adherence of patients, which largely rely on their correct understanding of pain and analgesics, was important but rarely involved in above studies. In our study, medication adherence of patients was evaluated and medicine education was conducted individually based on its initial score. The results showed that medicine education by $\mathrm{CP}$ changed patients' attitude to opioids and improved their adherence, making a better control of cancer pain. This finding suggested that medication education based on adherence is effective.

As for DRPs, Semerjian et al. suggested that at least 1 DRP was identified in $98.7 \%$ of the visits and more than $1 \mathrm{DRP}$ was identified in $71.6 \%$ patients (13). We recognized that the proportion was higher than that in ours. In the present study, at least 1 DRP was identified in $78.6 \%$ of patients and more than 1 DRP was identified in $51.5 \%$ of patients. It is possible that some of DRPs have been corrected by the CP during her 10-year pharmacy services in the ward. When regarding categories of DRPs, the most common DRP was inadequate pain control in our study, and the next two were no attention paid to $\mathrm{AE}$ or $\mathrm{AE}$ persist, and no opioids dosage reduction in time or opioids overdose. It is quite different from above findings, which showed medication refills needed was the most common one. This is attributed to a striking different medication situation of cancer pain between China and the United States. A Chinese scholar reported that the top three DRPs were nonadherence or missed doses (27.69\%), inappropriate opioids selection (22.56\%), and inappropriate dosage $(16.41 \%)$ (8). The proportion of inappropriate opioids selection was inconsistent with our study (5.3\%). It indicates that even in China, disparities exist between different medical centers.

Both Chen and Ding reported that standardization of opioids administration was significantly improved after the initiation of multidisciplinary interventions, including more frequent pain evaluation, more standardized dosing titration and so on $(7,14)$. We also found that the efficacy and safety in cancer pain pharmacotherapy were largely promoted through the pharmaceutical consultation and medication recommendation by CP. Besides, PI and majority of daily interferences significantly improved on the third day after the interventions and continued to the end of 28-day follow-up. Insufficient sample size might be the main limitation. Nearly half of patients had opioid-related $\mathrm{AEs}$, and the most common $\mathrm{AE}$ was constipation. Both the incidence of total $\mathrm{AE}$ and constipation in this study were lower than that observed in other researches $(7,15)$.

Strengths of this study mainly include the CP's active role in cancer pain treatment. Ten-year work in department of radiation oncology enabled her abundant experience on dealing with multiple links of cancer pain treatment. Her professional and meticulous pharmaceutical care has bridged the gap in doctors' treatment of cancer pain. The present study has preliminarily explored a model of comprehensive pharmaceutical service in cancer pain patients and proved its positive effect in clinical practice, which can be extrapolated to other centers. Furthermore, due to the combination of multiple drugs, patients with cancer pain are at a significant risk of DRPs, which cause or contribute to inadequate pain control or possible AEs, making it urgent to resolve. As we reported, DRPs occurred in the course of cancer pain treatment but could have been ameliorated with CP's interventions.

Several limitations need to be considered. Firstly, no control group was involved. Thus, we compared our outcomes with those in similar programs in China and other countries. Further studies included control groups are necessary to be conducted. Secondly, insufficient sample size was another limitation in this preliminary trial. Thus, this promising pharmacy service model must be validated with a larger population in subsequent studies. Thirdly, the followup period was relatively short, and more studies with longterm follow-up are needed to evaluate long-term impact of CPs in management of cancer pain pharmacotherapy.

\section{Conclusions}

Interventions by $\mathrm{CPs}$ in patients with cancer pain were efficacious in improving medication adherence and pain relief, as well as reducing incidence of AEs. Therefore, integration of comprehensive clinical pharmacy services into management of cancer pain related pharmacotherapy is a promising model that should be replicated in as many medical centers as possible.

\section{Acknowledgments}

The authors sincerely thank the physicians, nurses and administrators in the treatment team.

Funding: This study was supported by Research Funds 
of Shanghai Health and Family Planning commission (20184Y0022), Cultivation fund of clinical research of Renji hospital (PY2018-III-06), Clinical Pharmacy Innovation Research Institute of Shanghai Jiao Tong University School of Medicine (CXYJY2019ZD001, CXYJY2019QN004) and Program for Key but Weak Disciplines of Shanghai Municipal Commission of Health and Family Planning (2016ZB0304).

\section{Footnote}

Reporting Checklist: The authors have completed the STROBE reporting checklist. Available at http://dx.doi. org/10.21037/apm-20-627

Data Sharing Statement: Available at http://dx.doi. org/10.21037/apm-20-627

Peer Review File: Available at http://dx.doi.org/10.21037/ apm-20-627

Conflicts of Interest: All authors have completed the ICMJE uniform disclosure form (available at http://dx.doi. org/10.21037/apm-20-627). The authors have no conflicts of interest to declare.

Ethical Statement: The authors are accountable for all aspects of the work in ensuring that questions related to the accuracy or integrity of any part of the work are appropriately investigated and resolved. The study was conducted in accordance with the Declaration of Helsinki (as revised in 2013). This study was approved by Ethics Committee of Renji Hospital, School of Medicine, Shanghai Jiaotong University (KY2020-036). Written informed consent was obtained from the patients for publication of this study.

Open Access Statement: This is an Open Access article distributed in accordance with the Creative Commons Attribution-NonCommercial-NoDerivs 4.0 International License (CC BY-NC-ND 4.0), which permits the noncommercial replication and distribution of the article with the strict proviso that no changes or edits are made and the original work is properly cited (including links to both the formal publication through the relevant DOI and the license). See: https://creativecommons.org/licenses/by-nc-nd/4.0/.

\section{References}

1. Chen $\mathrm{W}$, Zheng R, Baade PD, et al. Cancer statistics in China, 2015. CA Cancer J Clin 2016;66:115-32.

2. Portenoy RK, Ahmed E. Principles of opioid use in cancer pain. J Clin Oncol 2014;32:1662-70.

3. Kwon JH. Overcoming barriers in cancer pain management. J Clin Oncol 2014;32:1727-33.

4. Scarborough BM, Smith CB. Optimal pain management for patients with cancer in the modern era. CA Cancer J Clin 2018;68:182-96.

5. Xia Z. Cancer pain management in China: current status and practice implications based on the ACHEON survey. J Pain Res 2017;10:1943-52.

6. Zhai XB, Gu ZC, Liu XY. Clinical pharmacist intervention reduces mortality in patients with acute myocardial infarction: a propensity score matched analysis. Eur J Hosp Pharm 2019;26:248-52.

7. Chen J, Lu XY, Wang WJ, et al. Impact of a clinical pharmacist-led guidance team on cancer pain therapy in China: a prospective multicenter cohort study. J Pain Symptom Manage 2014;48:500-9.

8. Liu J, Wang C, Chen X, et al. Evaluation of pharmacist interventions as part of a multidisciplinary cancer pain management team in a Chinese academic medical center. J Am Pharm Assoc (2003) 2020;60:76-80.

9. Tsai YF, Huang WC, Cho SF, et al. Side effects and medication adherence of tyrosine kinase inhibitors for patients with chronic myeloid leukemia in Taiwan. Medicine (Baltimore) 2018;97:e11322.

10. National Comprehensive Cancer Network Adult Cancer Pain Guidelines (version 1.2018). Available online: https:// www.nccn.org/professionals/physician_gls/default. aspx\#supportive

11. Wang $\mathrm{Y}$, Huang $\mathrm{H}$, Zeng $\mathrm{Y}$, et al. Pharmacist-led medication education in cancer pain control: a multicentre randomized controlled study in Guangzhou, China. J Int Med Res 2013;41:1462-72.

12. Edwards Z, Ziegler L, Craigs C, et al. Pharmacist educational interventions for cancer pain management: a systematic review and meta-analysis. Int J Pharm Pract 2019;27:336-45.

13. Semerjian M, Durham MJ, Mirzaian E, et al. Clinical Pharmacy Services in a Multidisciplinary Specialty Pain Clinic. Pain Pract 2019;19:303-9.

14. Ding $\mathrm{H}$, Zheng $\mathrm{X}$, Kong $\mathrm{S}$, et al. Multidisciplinary intervention incorporating pharmacists in management of 
opioid-naive patients with moderate to severe cancer pain. Eur J Cancer Care (Engl) 2020;29:e13225.

15. McNicol E, Horowicz-Mehler N, Fisk RA, et al.

Cite this article as: $\mathrm{Su} \mathrm{YJ}$, Yan YD, Wang WJ, Xu T, Gu ZC, Bai YR, Lin HW. Preliminary exploration on the role of clinical pharmacists in cancer pain pharmacotherapy. Ann Palliat Med 2020;9(5):3070-3077. doi: 10.21037/apm-20-627
Management of opioid side effects in cancer-related and chronic noncancer pain: a systematic review. J Pain 2003;4:231-56. 